\title{
Y-chromosome phylogeny in the evolutionary net of chamois (genus Rupicapra)
}

Trinidad Pérez ${ }^{1}$, Sabine E Hammer ${ }^{2}$, Jesús Albornoz ${ }^{1}$ and Ana Domínguez ${ }^{1 *}$

\begin{abstract}
Background: The chamois, distributed over most of the medium to high altitude mountain ranges of southern Eurasia, provides an excellent model for exploring the effects of historical and evolutionary events on diversification. Populations have been grouped into two species, Rupicapra pyrenaica from southwestern Europe and $R$. rupicapra from eastern Europe. The study of matrilineal mitochondrial DNA ( $m t D N A)$ and biparentally inherited microsatellites showed that the two species are paraphyletic and indicated alternate events of population contraction and dispersal-hybridization in the diversification of chamois. Here we investigate the pattern of variation of the Y-chromosome to obtain information on the patrilineal phylogenetic position of the genus Rupicapra and on the male-specific dispersal of chamois across Europe.

Results: We analyzed the Y-chromosome of 87 males covering the distribution range of the Rupicapra genus. We sequenced a fragment of the SRY gene promoter and characterized the male specific microsatellites UMN2303 and SRYM18. The SRY promoter sequences of two samples of Barbary sheep (Ammotragus lervia) were also determined and compared with the sequences of Bovidae available in the GenBank. Phylogenetic analysis of the alignment showed the clustering of Rupicapra with Capra and the Ammotragus sequence obtained in this study, different from the previously reported sequence of Ammotragus which groups with Ovis. Within Rupicapra, the combined data define 10 Y-chromosome haplotypes forming two haplogroups, which concur with taxonomic classification, instead of the three clades formed for mtDNA and nuclear microsatellites. The variation shows a west-to-east geographical cline of ancestral to derived alleles.

Conclusions: The phylogeny of the SRY-promoter shows an association between Rupicapra and Capra. The position of Ammotragus needs a reinvestigation. The study of ancestral and derived characters in the $Y$ chromosome suggests that, contrary to the presumed Asian origin, the paternal lineage of chamois originated in the Mediterranean, most probably in the Iberian Peninsula, and dispersed eastwards through serial funding events during the glacial-interglacial cycles of the Quaternary. The diversity of Y-chromosomes in chamois is very low. The differences in patterns of variation among Y-chromosome, mtDNA and biparental microsatellites reflect the evolutionary characteristics of the different markers as well as the effects of sex-biased dispersal and species phylogeography.
\end{abstract}

\section{Background}

Phylogenetic relationships within and between animal species often depend on the markers studied, as different genes might have different modes of transmission and different histories [1-3]. In addition, hybridization can result in discordant phylogenies between markers. Increasing evidence points to a contribution of reticulate

\footnotetext{
* Correspondence: sanjurjo@uniovi.es

'Departamento de Biología Funcional, Genética, Universidad de Oviedo,

Julián Clavería 6, 33006 Oviedo, Spain

Full list of author information is available at the end of the article
}

evolution to the speciation process [4-7]. In this context, information on the phylogenies of different markers for closely related species and subspecies is important to the study of processes underlying speciation [8].

The study of chamois (Rupicapra spp.) allows exploring the effect of historical and evolutionary events on diversification. It is distributed over most of the medium to high altitude mountain ranges of southern Eurasia (Figure 1). At present, chamois populations are classified into two species, $R$. pyrenaica and $R$. rupicapra [9], on the basis of morphological and behavioral characters:

C Biomed Central

(C) 2011 Pérez et al; licensee BioMed Central Ltd. This is an Open Access article distributed under the terms of the Creative Commons Attribution License (http://creativecommons.org/licenses/by/2.0), which permits unrestricted use, distribution, and reproduction in any medium, provided the original work is properly cited. 


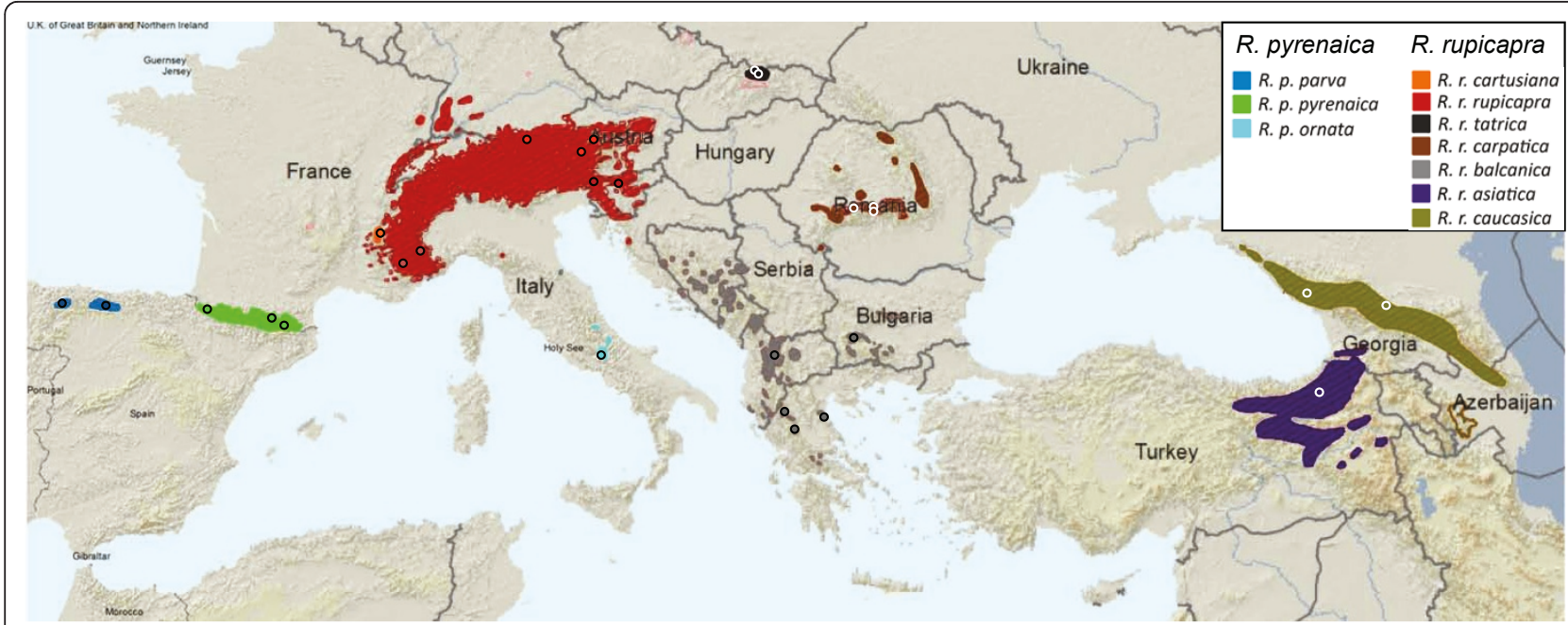

Figure 1 Geographic distribution of the subspecies of the genus Rupicapra. Sampling sites are indicated by circles. The map was modified from the distribution map on the IUCN Red List [73].

Rupicapra pyrenaica (with the subspecies parva, pyrenaica and ornata) from southwestern Europe, and $R$. rupicapra (with the subspecies cartusiana, rupicapra, tatrica, carpatica, balcanica, asiatica and caucasica) from central and southeastern Europe and western Asia [10]. Analysis of genetic variation in a limited number of subspecies for allozyme loci [11], minisatellites [12], RFLPs of mitochondrial DNA [13] and the major histocompatibility complex $[14,15]$ provided some support for this classification. However, the nominal species are paraphyletic for mtDNA [16,17].

The Quaternary glacial ages probably had a major effect on the phylogeography and evolution of the genus Rupicapra, as it did on other animals in Eurasia [18-21]. The Rupicaprini are thought to have originated in Asia during the Miocene period and the sudden appearance of Rupicapra fossils in Europe during the middle Pleistocene age has been interpreted as resulting from immigration from the east during a cold climatic phase [21]. In contrast with the fossil record, the divergence between the main mtDNA clades has been estimated around 1.5-3 mya [16,17,22-25] but this cannot be directly assumed to be the divergence time between species. The mitochondrial phylogeny showed three main lineages, originating during the Early Pleistocene $[16,17]$. Nuclear microsatellite genotypes formed three clearly defined groups as well; however those groups did not exactly match the mitochondrial lineages but are closer to morphology and taxonomic classification. The phylogeographic patterns suggest an evolutionary history with range contractions and expansions related to climatic oscillations during the Quaternary period and reflect a major effect of the Alpine barrier on west-east differentiation. The contrasting phylogenies of mtDNA and nuclear microsatellites for populations of Chartreuse and the western Alps indicated events of range overlap and hybridization among highly divergent lineages in the central area of the distribution. Both markers showed differentiation between all pairs of populations $[16,17,26]$ and a geographic signature in the distribution of variability, suggesting that differentiation occurred without major migrations.

To further elucidate the processes leading to the origin and diversification of Rupicapra, we studied the Ychromosome. The Y-chromosome is paternally inherited and does not undergo recombination at meiosis, providing a marker to study male dispersal [27-29]. The study has the dual purpose of studying the patrilineal phylogenetic position of chamois, compared with Capra, Ovis and Ammotragus, and the male dispersal within the genus Rupicapra. We present the analysis of a sequence of a fragment of the SRY gene promoter together with two male-specific microsatellites UMN2303 and SRYM18, in a sample set of 87 males, 40 of $R$. pyrenaica and 47 from $R$. rupricapra, which covers the entire distribution range of chamois. Comparison of the geographic distribution of male-specific markers with mtDNA lineages (defining matrilines) and autosomal markers (biparentally inherited) allows us to follow the evolutionary history of Rupicapra in the context of the climatic oscillations of the Pleistocene age.

\section{Results}

\section{SRY promoter sequences}

We have amplified and sequenced 569 nucleotides corresponding to a fragment of the SRY gene promoter from 52 male chamois, 24 of the subspecies $R$. pyrenaica (14 parva, 6 pyrenaica and 4 ornata) and 28 of $R$. 
rupicapra (5 cartusiana, 6 rupicapraW, 6 rupicapra $C E$, 3 tatrica, 3 carpatica, 2 balcanica, 1 asiatica and 2 caucasica). The alignment resulted in only two haplotypes, one in $R$. pyrenaica and the other in $R$. rupicapra. These haplotypes differ only in one nucleotide (site 267 in our alignment), which is $\mathrm{A}$ in the haplotype pyrenaica and $\mathrm{G}$ in rupicapra.

To investigate the evolutionary history of the Y-chromosome of Rupicapra, the two haplotypes were aligned with the sequences of other Bovidae available in the GenBank, Ammotragus lervia, Capra hircus, Ovis aries and Bos taurus (see Table 1). In addition, two individual Ammotragus lervia have been sequenced in our laboratory and both had identical sequence with a deletion of 44 nucleotides with respect to the rest of Bovidae. The aligned dataset contains 531 nucleotides (481 nt, indels excluded) with 78 variable sites of which 34 are fixed and 44 are variable among Caprinae. The phylogenetic relationships were studied using Neighbor-Joining, Maximum Likelihood, Maximum Parsimony, or Bayesian approaches under different models of nucleotide substitution, either the simple model of Jukes-Cantor or the substitution model that describes better the substitution pattern of the dataset, a Tamura 3-parameter model [30] with non-uniformity of evolutionary rate among sites $(\mathrm{T} 92+\mathrm{G})$. The three parameters were nucleotide frequencies 0.3392 for $\mathrm{A}$ and $\mathrm{T}, 0.1608$ for $\mathrm{C}$ and $\mathrm{G}, \mathrm{Ts} / \mathrm{Tv}$ ratio: 1.6281 and rate heterogeneity: 0.4762 . For the construction of the Bayesian tree, the model of nucleotide substitution was HKI+G (also appropriate to describe the observed substitution pattern since it has the second lowest BIC score obtained with MEGA) and the parameters were obtained by the program BEAST itself. There were 36 parsimony-informative sites. Model-free Parsimony Analysis performed with MEGA led to three equally parsimonious trees with a total length of 87 steps. The different methods of tree construction all led to topologies

Table 1 References of SRY promoter sequences previously available and obtained in this work

\begin{tabular}{lll}
\hline Species & GenBank Accession No & Reference \\
\hline Bos taurus & EU581861.1 & {$[74]$} \\
Ovis aries & EU938044.1 & {$[35]$} \\
Ovis aries musimon & EU938022.1 & {$[35]$} \\
Ovis ammon & EU938024.1 & {$[35]$} \\
Ovis canadensis & EU938032.1 & {$[35]$} \\
Ovis vignei & EU938028.1 & {$[35]$} \\
Capra hircus 1 & EU581862.1 & {$[74]$} \\
Capra hircus 2 & D82963.1 & {$[75]$} \\
Ammotragus lervia 1 & EU938019.1 & {$[35]$} \\
Ammotragus lervia 2 & JN547784 & Present study \\
Rupicapra pyrenaica & JN547785 & Present study \\
Rupicapra rupicapra & JN547786 & Present study \\
\hline
\end{tabular}

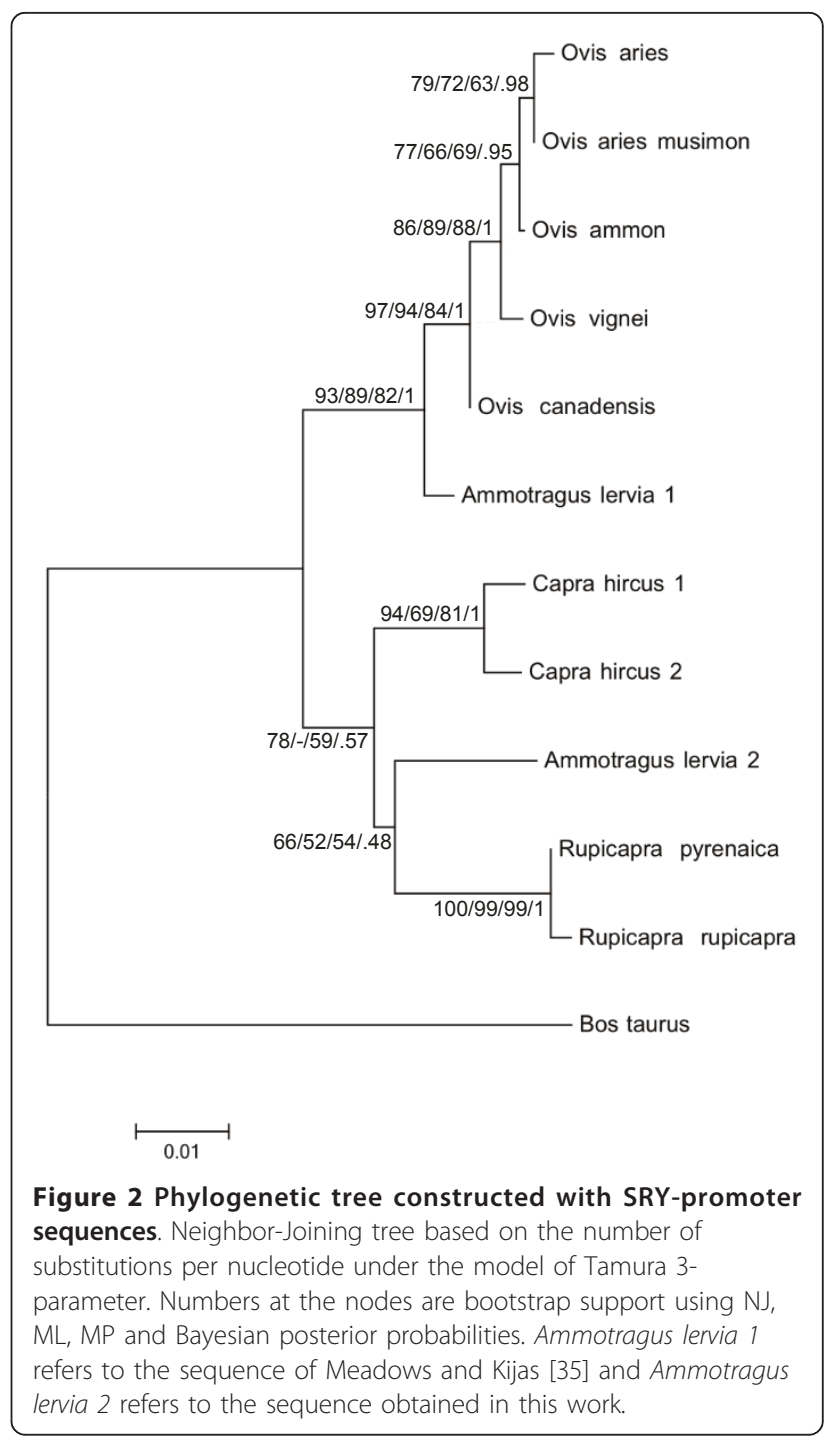

with two main well supported nodes (Figure 2), one grouping Ovis with the published sequences of Ammotragus and the other grouping Rupicapra, Capra and the sequence of Ammotragus obtained in this work. The relationships within this second group varied, depending on the method used for tree construction, and they were poorly supported. All the 10 different Bovidae sequences present, like pyrenaica, A in site 267 in our alignment, suggesting that this is the ancestral haplotype.

The estimated divergence time of the two Rupicapra haplotypes from the SRY promoter sequence was 655 kya (95\% CI: 10-1,611). The mean substitution rate per nucleotide calculated with TRACER from the MCMC samples was $2.09 \mathrm{E}-3 \pm 1.08 \mathrm{E}-5$ per million years.

\section{Y-chromosome microsatellites}

Out of 14 microsatellite markers tested, only UMN2303 and SRYM18 produced male-specific products. 
Amplification from 87 males, 40 of $R$. pyrenaica and 47 from $R$. rupricapra, revealed two different length fragments for UMN2303 and seven for SRYM18 (Table 2). For each microsatellite, fragments within each length variant were further analyzed by cloning and sequencing, and the architecture was compared with their homologous loci in other Bovidae. The UMN2303 repeated motif was found to be $[\text { TTTTG }]_{\mathrm{n}}$ differing from the repeat $[\mathrm{TG}]_{\mathrm{n}}$ reported in Bos taurus. Rupicapra pyrenaica presented two alleles, 125 and 130, differing in one repetition, and $R$. rupicapra was monomorphic, with only the 125 allele. The microsatellite SRYM18 lacks the pentanucleotide [TTTTG] and the dinucleotide [TG] motifs common in sheep [31] and instead presents a trinucleotide $[\mathrm{TTC}]_{\mathrm{m}}$ and a mononucleotide $[\mathrm{T}]_{\mathrm{n}}$ motifs. Rupicapra pyrenaica haplotypes were $[\mathrm{TTC}]_{\mathrm{m}} \mathrm{A}[\mathrm{T}]_{\mathrm{n}}$ and $R$. rupicapra haplotypes were $[\mathrm{TTC}]_{\mathrm{m}}[\mathrm{T}]_{\mathrm{n}}$, these two structures were reported in Ammotragus lerviae (Acc. $\mathrm{N}^{\circ} \mathrm{DQ} 272449$ ) and Ovis aries breed Balami (Acc. N DQ272459.1), respectively. Combinations of variation in number of repeats in both motifs in $R$. rupicapra resulted in homoplasy, where PCR products with the same size had different sequence architecture. The trinucleotide motif, $[\mathrm{TTC}]_{\mathrm{m}}$, was polymorphic in the species $R$. rupicapra but not in $R$. pyrenaica while the mononucleotide motif was polymorphic in both subspecies (Table 2).

\section{Network of $Y$ haplotypes}

Altogether, ten haplotypes could be differentiated in the Rupicapra genus (Table 2). Total Y-chromosome haplotype diversity was 0.82 with on average one distinct haplotype over 8.7 individuals (87/10). Three private haplotypes were found in west chamois $R$. pyrenaica, giving a haplotypic diversity of $51.50 \%$ and the other seven haplotypes were private of the east chamois $R$. rupicapra with a diversity of $74.69 \%$.

The network of haplotypes (Figure 3 ) revealed two haplogroups that concur with the taxonomy of chamois. These two clades are separated by two nucleotide substitutions (one in the SRY promoter and one in the SRYM18 microsatellite) and a mean distance of 2.11 microsatellite repeats. The connections between haplotypes show a strong geographic signal with links always between neighboring populations. The same network is obtained whether or not the nucleotide substitutions are included.

\section{Discussion}

Analysis of chromosome $\mathrm{Y}$ presented a pattern of variation different from the one obtained from either mitochondrial or biparental nuclear DNA, both on diversity as well as on the spread and geographic boundaries of dispersion. However, the east/west phylogeographic

Table 2 Haplotypes of the male-specific region of the Y-chromosome of Rupicapra (generated through the combination of the sequence of the SRY promoter and microsatellites UMN2303 and SRYM18), and frequencies across subspecies

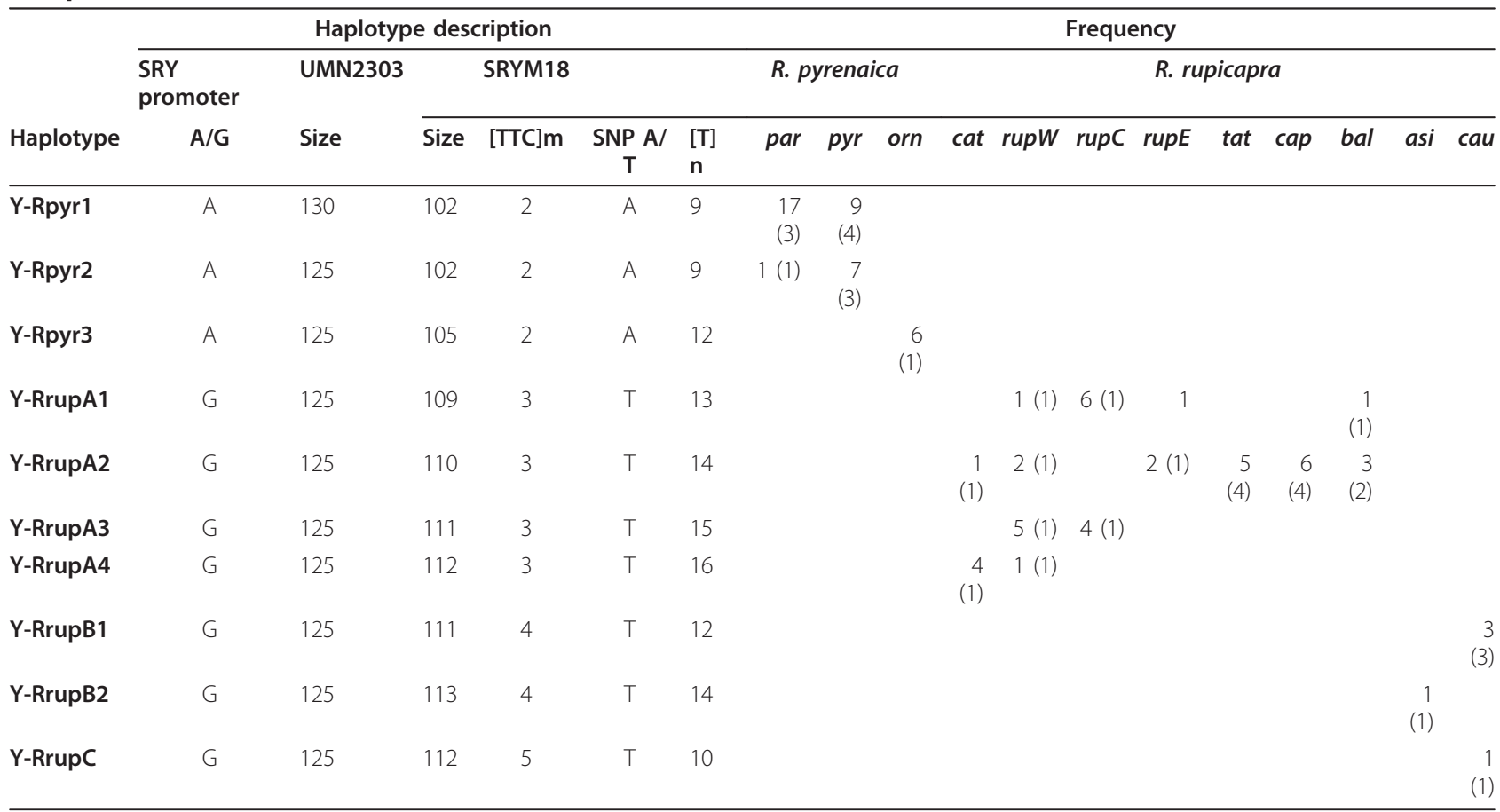

The abbreviated name of the subspecies are: par, parva; pyr, pyrenaica; orn, ornata; cat, cartusiana; rupW, rupicapraW; rupC, rupicapraC; rupE, rupicapraE; tat, tatrica; cap, carpatica; bal, balcanica; asi, asiatica; and cau, caucasica. Number of samples sequenced for SRYM18 are given in parenthesis. 


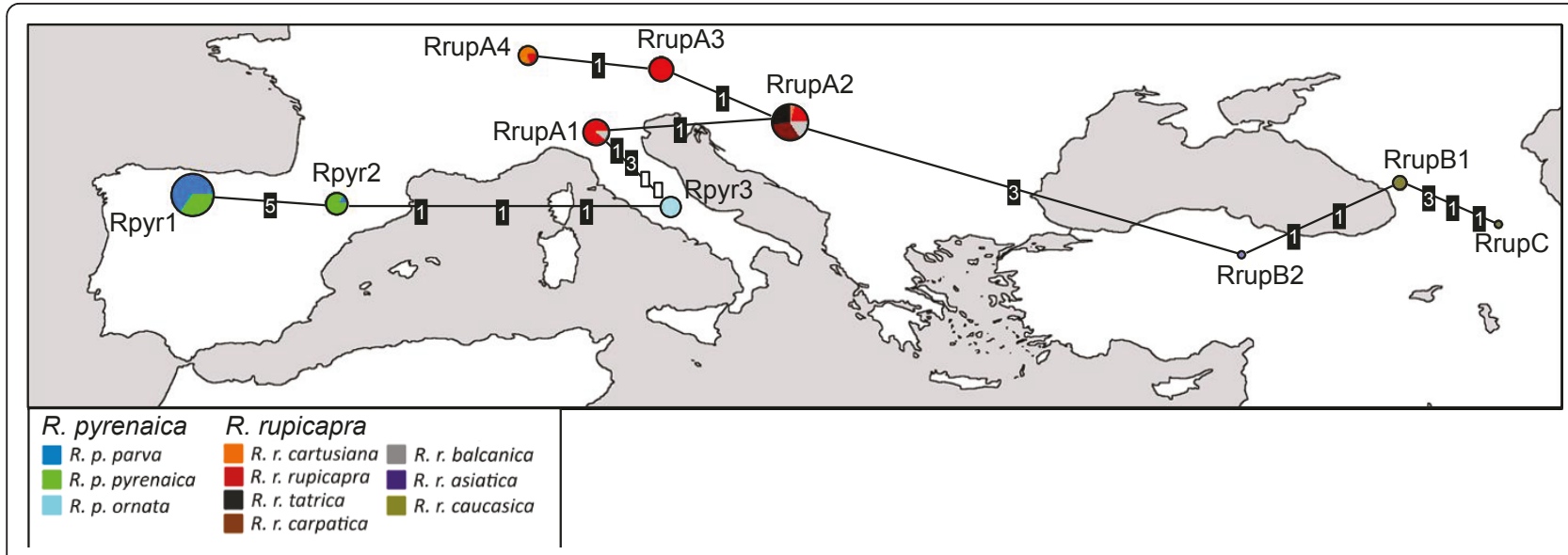

Figure 3 Network of Y-chromosome haplotypes. Median-joining network for the Y-chromosome haplotypes constructed using variation at the SRY promoter sequence and at the microsatellites UMN2303 (number of pentanucleotide repeats) and SRYM18 (one SNP, number of trinucleotide repeats and number of mononucleotide repeats). The size of pie areas corresponds to haplotypic frequencies and the proportion accounted for by the different subspecies is represented in different colors as in Figure 1. Different types of mutations in each branch are represented by different symbols (white square: SNP; black squares with a number inside: microsatellites with mononucleotide [1], trinucleotide [3] and pentanucleotide [5] motifs). The network is represented over a map according to the approximate geographical origin of the haplotypes. Branch lengths are not scaled.

signal in the distribution of haplotypes is present again and once more the suture zone places in the Alps. It is remarkable that, contrary to mtDNA and autosomal microsatellites that formed three clades (although not exactly concordant), the variation for the Y-chromosome conforms to the two species currently accepted, Rupicapra pyrenaica and $R$. rupicapra. This could explain the concurrence of coat patterns, cranial morphometry and several courtship behavioral patterns in Iberian and Apennine chamois $[10,21,32]$ that remained unexplained from the study of mtDNA and nuclear microsatellites [33].

\section{Phylogenetic relationships between chamois and other caprini}

The phylogeny of SRY promoter shows an association between Rupicapra, Capra and the Ammotragus sequence obtained in this work (Ammotragus 2). This association concurs with the relationships revealed from the study of the complete mitochondrial genome [34]. Previously, Meadows and Kijas [35] reported a very close relation between the SRY promoter of Ammotragus (Ammotragus lervia 1 in Table 1 and Figure 2) and Ovis, but we found 15 differences (3.33\%) between this previously reported sequence and the new sequences produced in our laboratory (Ammotragus lervia 2 in Table 1 and Figure 2). This large difference is not expected between two individuals of the same species. In contrast, the reported sequence of Ammotragus presents only 4 differences $(0.83 \%)$ with Ovis canadensis. Our sequence has been obtained from good quality samples (muscle) from two specimens (both repeated twice) with identical results. So, we think that the sequence reported by Meadows and Kijas could be contaminated with DNA of Ovis canadensis. An alternative interpretation to take into account is the polyphyly of Ammotragus. The affinities of Ammotragus with either Capra or Ovis have been widely discussed in the literature as it exhibits a particular combination of goat-like and sheeplike characters [36]. The structure of the microsatellite SRYM18 of west chamois is identical to Ammotragus and different to most Ovis, but the African breed Balami of $O$. aries shares the repeat structure with Ammotragus and Rupicapra. This observation had lead Meadows and Kijas [31] to hypothesize the possible gene flow from Barbary to domestic sheep. The observed similarities between Ammotragus lerviae and the genus Rupicapra and the apparent spread of male chamois south to north reopen the question of the possible position of Ammotragus as an ancestor of the Caprinae [37]. It can also be noted that Rupicapra and Ammotragus have similar karyotype with 58 chromosomes [38]. Additional studies of Y-chromosome phylogenies of Caprinae could offer very important information to clarify this issue.

\section{Patrilineal phylogeography of chamois}

When comparing the phylogenetic trees based on mtDNA or the sequences of the SRY promoter (Figure 4), a clear difference emerges. All the Rupicapra belong to one unique clade for the SRY promoter while three, well differentiated, clades formed for mtDNA. The observed number of substitutions per nucleotide between the pairs of species Ovis-Rupicapra, CapraRupicapra and Ovis-Capra for the sequences of mtDNA 


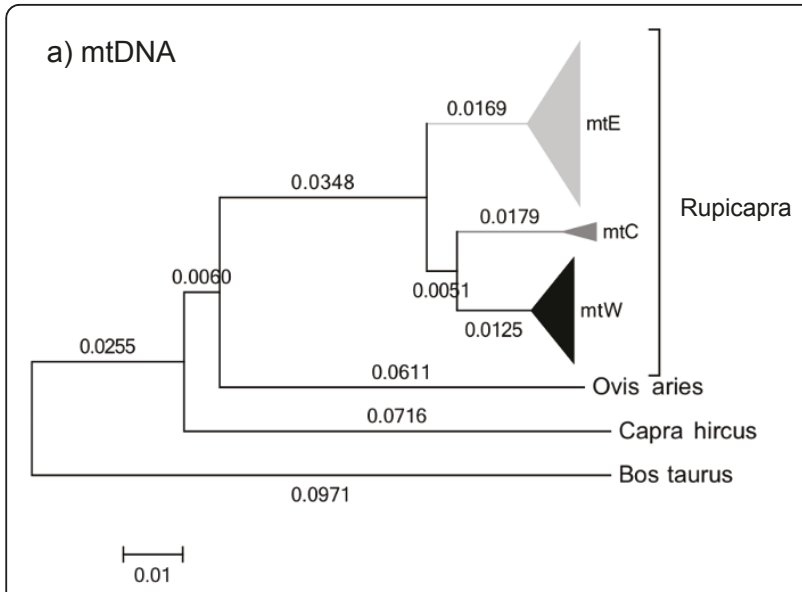

b) SRY

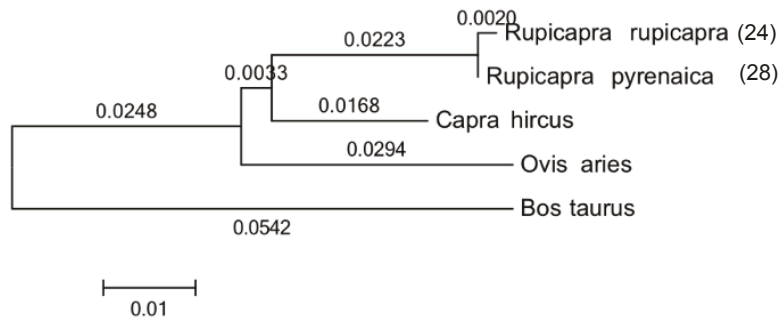

Figure 4 Comparison of mitochondrial DNA and SRY-promoter phylogenies on chamois. Neighbor-Joining trees under Jukes-Cantor showing the relationships among chamois and the outgroups Ovis aries, Capra hircus and Bos taurus. a) Tree constructed with a combined sequence of 1708 nucleotides of mtDNA [17]. Sequences in clades mtW, Clade mtC and Clade mtE, in black, grey and white respectively, were collapsed. b) Tree constructed with a sequence of 531 nucleotides of the SRY promoter. For both trees the number of individuals in the external branches is indicated in parentheses.

in our former study [17] were $0.1125,0.1264$ and 0.1186 respectively to be compared with $0.0520,0.0346$ and 0.0489 substitutions per nucleotide respectively for the SRY promoter sequence. The distance between pairs of species for mtDNA is about two or three times that of the SRY promoter, consistent with observations in other mammals including humans [39-42]. The level of differentiation among Y-chromosomes in chamois is remarkably low. The haplogroups Y-Rpyr and Y-Rrup within the Rupicapra genus differ by one single nucleotide, leading to an estimated average number of substitutions per nucleotide of 0.0019 that is 24.6 times lower than the average distance between the three clades of mtDNA (0.0468). The time of divergence between the SRY haplotypes estimated from the phylogenetic tree places the split $655 \mathrm{kya}$, in the middle of the Pleistocene. Thus, all modern chamois seem to descend of one very young male lineage. The low diversity in the number of microsatellite repeats, both between species and within species, compared with the $\mathrm{Y}$-specific evolutionary mutation rate of $2.6 \times 10^{-4}$ mutations per generation [43], gives further support to this interpretation. Thereafter, our data suggest that the divergence of Y-chromosomal variants took place well after the divergence of mtDNA lineages $[17,22,44]$, in a period compatible with the sudden appearance of Rupicapra fossils in Europe [21].

The examination of the Y-chromosome network of haplotypes offers insight into the patrilineal history of dispersion. There is an association between the network of haplotypes and geography (see Figure 3 ) with a westeast cline of ancestral to derived chromosomes and the signature of the Alpine barrier. Attending to the SNP in the SRY promoter it can be noted that the haplogroup Y-Rpyr presents the ancestral plesiomorph state (A in position 267 in our alignment), shared by all the other species in the phylogeny, while the haplogroup Y-Rrup has the derived state $G$ (transition $A>G$ ). Regarding the SNP in the microsatellite SRYM18, Y-Rpyr presents the nucleotide A like Ammotragus, hence it could be assumed to represent the ancestral state, while Y-Rrup presents $\mathrm{T}$ (transversion $\mathrm{A}>\mathrm{T}$ ). From here we propose that one of the haplotypes of the Y-pyr group represents the ancestral state. The pattern of microsatellite variation within each haplogroup also supports this interpretation as explained below. First, let us recall that the mutation profiles of microsatellites depend on the size of the repeat motif, the rate of mutation of pentanucleotides is about half of that of trinucleotides, and mononucleotide repeats present a very high mutation rate [45]. Our data on pentanucleotide, trinucleotide and mononucleotide difference between $R$. pyrenatica and $R$. rupicapra in number of repeats $(0.65,1.13$ and 4.55 , respectively) concur with this observation. Hence, microsatellites with longer motifs retain a stronger phylogenetic signal than shorter ones. It has been shown that in microsatellites with few repetitions, mutation is biased towards increase in number of repeats $[46,47]$. Attending to the network of haplotypes, it can be seen that Rpyr2, sampled from $R . p$. pyrenaica and $R . p$. parva, presents the lowest number of repeats for all, the penta, the tri and the mononucleotide motifs, this is probably the closest to the basal haplotype. The number of repeats of the pentanucleotide microsatellite UMN2303 is variable only in western chamois, the allele 130 , with one additional repeat is found in $R . p$. 
pyrenaica and $R$. p. parva. The number of trinucleotide repeats and mononucleotide repeats of SRYM18 increases west to east. The mononucleotide motif seems to have suffered mutations forth and back in the eastmost populations.

We conclude that the patrilineal dispersion of Rupicapra was south-west to north-east. The cline of ancestral to derived chromosomes could be originated by serial funding events, probably related to the glacial-interglacial cycles of the Quaternary. Male dispersion could start from the Iberian Peninsula or from Italy (Figure 5) to the east and the two haplogroups differentiate one to each side of the Alps. The lower number of repeats points to haplotype Rpyr2 present in Iberia as the closest to the ancestor. Alternatively, the haplotype Rpyr3 in the center of the network and differing from Rpyr2 solely in the mononucleotide could be also a good candidate.

\section{Differences between markers in patterns of variation and the evolutionary history of chamois}

The discordant patterns of mtDNA and autosomal microsatellites that have been described in a previous study [17] indicated the occurrence of hybridization among divergent lineages of chamois in the central area of the distribution. Chromosome Y data add complexity to this mosaic; the geographical sorting of variation for the different kind of marker is summarized in Figure 5. The three kinds of markers have specific evolutionary characteristics that need to be taken into account to explain differing variation patterns [27]. These differences are on (1) mutation rates, (2) selection, (3) effective population size and (4) dispersal. (1) Being the Y a male-specific chromosome, and given that the mutation rate is higher in males than in females [48], we would expect higher divergence for $\mathrm{Y}$-chromosome than for autosomal microsatellites; our data are contrary to this expectation. (2) Positive selection could act on the whole Y-chromosome and, given that the Y-chromosome does not recombine, it will be selected as a block in a selective sweep with the consequence of homogenization [28]. In addition, the lack of recombination must be responsible for the effect known as Muller's ratchet, the random accumulation of deleterious mutations that cannot be removed by recombination $[49,50]$. Selection against these mutations further reduces variability. Selection has been proposed as the main factor to explain low levels of genetic variability in the Y-chromosome [51] and must contribute to the reduced variability among Y-chromosomes in chamois. A similar pattern was found in Ursidae where Y-chromosome genes have fewer substitutions than expected in external branches [41]. (3) The chromosome Y and mtDNA both have effective population size of one quarter of that of an autosome because they are haploid and transmitted by one sex, but the effective population size of Y-chromosome must be lower because the variance in number of descendants is larger in males than in females, especially in a polygynous species. Hence, the Y-chromosome is more prone to genetic drift effects. However, genetic drift cannot be invoked as the main cause for the $\mathrm{Y}$ chromosome phylogeography because in such case no such strong geographical signal would be expected. (4) Differences in male and female dispersal can be related to different patterns of variation for patrilineal, matrilineal and biparental markers. Recent migration of males could be responsible of the low Y-chromosome diversity observed.

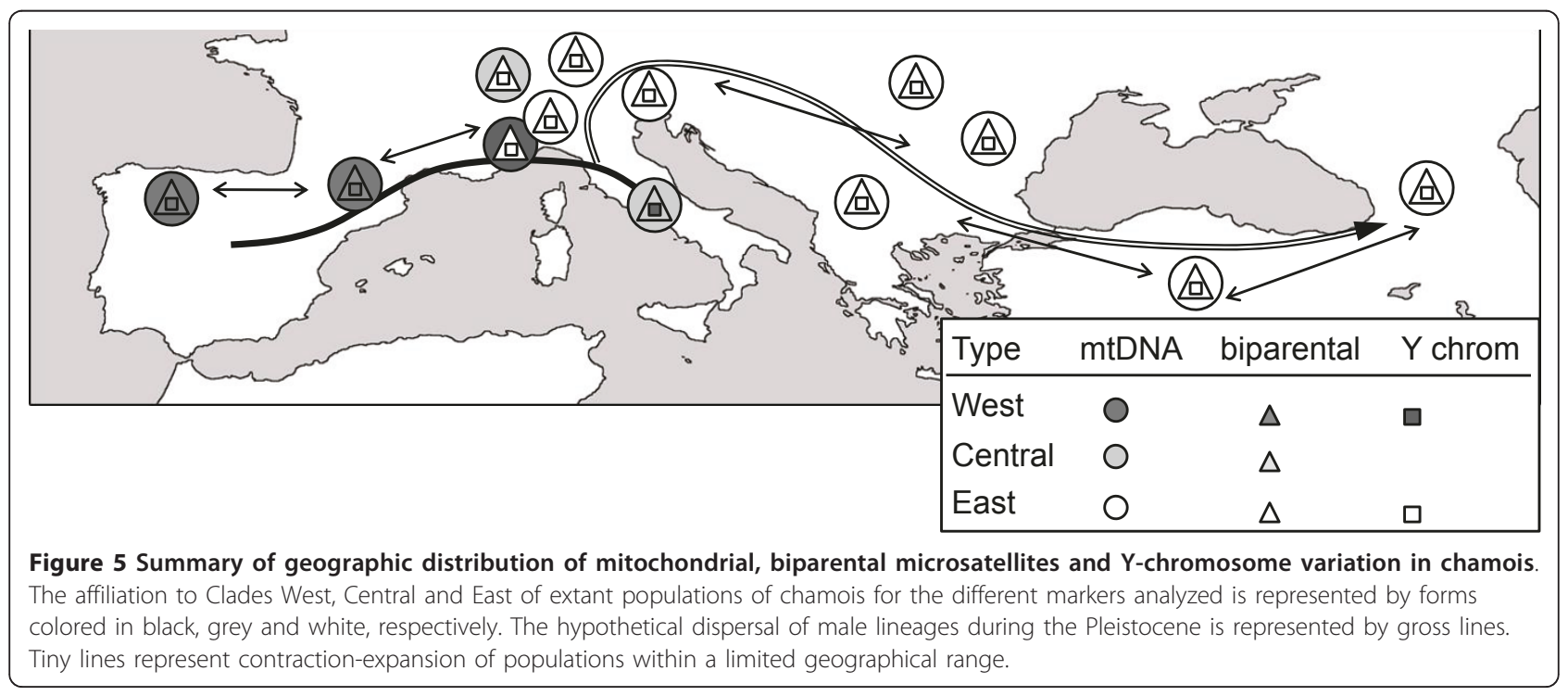


Taking into account the above considerations, together with the assumed Asiatic origin of Rupicapra and the similarity of the Y-chromosome of Rupicapra and Ammotragus, we propose two speculative hypotheses to explain the observed results: a) The Rupicapra genus has its origin in southern Europe and spread west to east. The differences in divergence between mtDNA and Y-chromosome are due to the particular evolutionary characteristics of the Y-chromosome, or b) males from an ancestral population highly differentiated from a second ancestral taxa present in Europe entered from the south, hybridizing and spreading westwards. Under the first hypothesis, the origin of the genus Rupicapra needs to be placed in south Europe from where it spread eastwards. This is contrary to the general belief that chamois, as caprines in general, originated in Asia [21] but, following Ropiquet and Hassanin [23], this hypothesis is not really supported by the fossil record. These authors propose that caprines originated in the Mediterranean islands during the Miocene and from here may have invaded Africa, Europe and Asia after the Tortonian salinity crisis (7.8-7.6 mya) or after the Messinian salinity crisis (6-5.3 mya). The similarity among Ychromosomes under this hypothesis must be attributed to male dispersal and female phylopatry, together with selective sweeps and purifying selection that acted over the chromosome as a whole. The second hypothesis is the hybridization between two ancestral Caprinae in the beginning of the evolutionary history of the genus Rupicapra. The hybridization between highly divergent lineages at speciation of Rupicapra had been previously hypothesized after the observation of a pseudogene of cytochrome $b$ from a highly divergent lineage in the nucleus of present day chamois [24,37,52]. An ancestral Caprinae related with Ammotragus could have reached the south Mediterranean, most probably the Iberian Peninsula, and hybridize with the ancestral Rupicapra female. The dispersal of species across the strait of Gibraltar during Pleistocene has been proposed for other vertebrates $[40,53,54]$ and has been related to the substantially lower sea level associated with major Pleistocene glaciations [40]. New male Y-chromosome lineages would have dispersed during the Pleistocene, replacing older ones. Hybridization, after secondary contact among related taxa, has been inferred in the evolution of the genus Capra [55] as well as in the evolution of some species of Bos [56] and has been recently observed between native and introduced species of Cervus in Scotland [57]. It can be added that hybrids from Ammotragus male and Capra female have been artificially obtained [58]. Many recent studies have shown contrasting phylogenies for different kinds of markers that lead to the view of hybridization as an important mechanism in the evolution of animal species $[5,7]$.
Our results on Y-chromosome reconcile the sudden occurrence of Rupicapra in the middle-late Pleistocene with the existence of very old mtDNA lineages. In addition, the proposed migration of chamois west to east can explain the reported more conservative features of $R$. pyrenaica [33]. The alternative hypotheses presented here can be tested in future research that includes the comparative study of chromosome $\mathrm{Y}$ in a broader representation of Caprinae and additional nuclear sequences of the different populations of Rupicapra to search for the signature of possible hybridizations.

\section{Conclusions}

The Y-chromosome is highly informative to follow the dispersal of populations of Caprinae. The phylogenetic analysis of the SRY gene promoter shows an association between Rupicapra, Capra and Ammotragus, not in agreement with a previous report, which grouped Ammotragus with Ovis. The structure of the SRYM18 microsatellite of Rupicapra is equal to Ammotragus and the African breed Balami of $O$. aries. The patrilineal relationships of Caprine deserve a reinvestigation. Within Rupicapra, the diversity of Y-chromosomes is very low. The combined data define $10 \mathrm{Y}$-chromosome haplotypes forming a west-to-east geographical cline of ancestral to derived alleles. Haplotypes form two haplogroups, which concur with taxonomic classification, instead of the three clades formed for mtDNA and biparental microsatellites. We propose that, contrary to the presumed Asian origin, the paternal lineage of chamois originated in the Mediterranean, most probably in the Iberian Peninsula, and dispersed eastwards through serial funding events during the glacial-interglacial cycles of the Quaternary. The differences in patterns of variation among Y-chromosome, mtDNA and biparental microsatellites reflect the evolutionary characteristics of the different markers, as well as the effects of sex-biased dispersal and species phylogeography.

\section{Methods}

\section{Sampling, DNA extraction and sex determination}

Samples of the 10 recognized subspecies of chamois were collected from 1992 until the present, covering the distribution range of the genus Rupicapra (see Figure 1) and have previously been analyzed for autosomal microsatellites and mtDNA $[16,17,26]$. For large populations, where hunting is allowed, samples were either of muscle or skin preserved in $96 \%$ ethanol by gamekeepers, or teeth from skulls sent to taxidermists. For protected populations, samples were obtained from animals found dead; tissues, as well as their conservation method, were diverse (hair, bone, salted skin and muscle in ethanol) and were sent by biologists. Two muscle samples of Barbary sheep (Ammotragus lervia) one from Sierra 
Espuña (Murcia, Spain) and the other from Caldera de Taburiente (La Palma, Canary Islands, Spain) were included.

Due to the different origin and type of the material included in this study, different methods of DNA isolation were used. DNA from bones or teeth was extracted by a method modified from Catanneo et al. [59] as described [26]. For soft tissue samples, DNA was extracted either with the phenol/chloroform method [60] using Chelex, following Estoup et al. [61] or using the 'DNeasy Tissue kit' (Qiagen, Hilden, Germany). Finally, 56 of the 215 samples were collected and the DNA was extracted in the laboratory of Vienna (Austria) following the protocol described in the Genetic Analysis Manual (LI-COR, Inc. 1999), followed by a standard phenol/chloroform extraction and DNA precipitation procedure [60].

We determined the sex of each individual sampled using the SE47/SE48 sex identification primers [62]. SE47/SE48 primers amplify one DNA fragment for females (indicating the presence of $\mathrm{X}$-chromosome) and two for males (indicating the presence of a X and a $\mathrm{Y}$ chromosome). Reactions were performed in a final volume of $20 \mu \mathrm{l}$ containing $2 \mu \mathrm{l}(\approx 40-70 \mathrm{ng})$ DNA, 0.5 $\mathrm{mM}$ of each primer, 1x PCR Buffer, $200 \mathrm{mM}$ of each dNTP, $2.5 \mathrm{mM} \mathrm{MgCl}_{2}$ and $1 \mathrm{U}$ of Taq DNA polymerase (Qiagen, Hilden, Germany). Amplification was carried out in PE GeneAmp PCR 9700 thermal cycler (Applied Biosystems, Foster City, CA) with an initial step of 5 min at $94^{\circ} \mathrm{C}, 35$ cycles of $30 \mathrm{~s}$ at $94^{\circ} \mathrm{C}, 30 \mathrm{~s}$ at $60^{\circ} \mathrm{C}$ and $1 \mathrm{~min}$ at $72^{\circ} \mathrm{C}$, followed by $5 \mathrm{~min}$ at $72^{\circ} \mathrm{C}$. PCR products were visualized on $2 \%$ agarose gels, pre-stained with ethidium bromide. We tested 222 samples, 135 were discarded either because they were from females (83 samples) or they did not amplify (52 samples), and 87 male samples, 40 from $R$. pyrenaica and 47 from $R$. rupicapra were used in the study of Y-chromosome.

\section{Chromosome $\mathrm{Y}$ genotyping}

A total of fourteen Y microsatellite loci and six Y-chromosome sequences were tested on chamois for amplification and male specificity (Pérez et al. in preparation). Only two microsatellites, one bovine UMN2303 [63] and one ovine SRYM18 [31] and one male specific sequence, a fragment of the 5'-promoter of the sex determining region $Y$ (SRY) gene amplified with primers designed from Ovis [31], gave consistent male-specific products and were chosen for further analysis.

The microsatellite loci UMN2303 and SRYM18 were amplified in $20 \mu \mathrm{l}$ PCR reactions containing $2 \mu \mathrm{l}(\approx 40$ $70 \mathrm{ng})$ DNA, $0.5 \mathrm{mM}$ of each primer, $2.5 \mathrm{mM} \mathrm{MgCl}_{2}$, 1x PCR Buffer, $200 \mathrm{mM}$ of each dNTP, $2.5 \mathrm{mM} \mathrm{MgCl}_{2}$ and $1 \mathrm{U}$ of Taq DNA polymerase (Qiagen, Hilden, Germany). The annealing temperature was $55^{\circ} \mathrm{C}$ for
SRYM18 and $58^{\circ} \mathrm{C}$ for UMN2303. Fluorescently labeled forward primers were used. Amplification was carried out using the PE GeneAmp PCR 9700 (Applied Biosystems). PCR products were checked in a $2 \%$ agarose gel and the product diluted up to 100 -fold depending on the signal intensity. One microlitre of the dilution was added to a $12 \mu \mathrm{l}$ mix of formamide and ROX 400HD (12:0.2) and loaded on an automatic sequencer ABI310 (Applied Biosystems). Microsatellite patterns were examined both visually and using GENESCAN ANALYSIS 3.1 and GENOTYPER 2.5 software (Applied Biosystems).

Sequencing was carried out either cloning the PCR products (UMN2303 and SRYM18 STR loci) or directly (SRY promoter). When cloning was needed, products of amplification were purified using GFX PCR DNA and Gel Band Purification Kit (Amersham Biosciences, Buckinghamshire, UK), and they were directly cloned into the PMOSBlue vector (Amersham Biosciences) and transformed into MOSBlue competent cells according to the supplier's specification. Clones were screened for inserts of the expected size by PCR amplification with the universal primers M13 and T7. For selected clones, plasmid DNA was prepared for sequencing following Sambrock et al. [64]. For direct sequencing PCR-amplified products were purified with the Exo-SAP-IT kit (USB Corporation, Cleveland, $\mathrm{OH}$ ). Sequencing reactions were performed for both strands using the appropriate primers and the BigDye Terminator v3.1 Cycle Sequencing Kit (Applied Biosystems). Sequencing products were purified with isopropanol precipitation and sequenced in an ABI 310 Genetic Analyzer (Applied Biosystems). The raw sequence data were analyzed using the ABI Prism DNA Sequencer Analysis software v3.4.1. After the occasional observation of differences among the number of mononucleotide repeats in the sequences from SRYM18, the determination of allele size and sequence was repeated for a subset of 16 samples. The results on allele size were always repeatable while the sequences presented errors due to slippage. Hence, the number of mononucleotide repeats was adjusted according to allele size.

\section{Phylogenetic analysis of SRY promoter sequences}

The SRY promoter sequences were manually aligned and edited using MEGA5 [65]. The two different haplotypes identified in Rupicapra plus the sequence of Ammpotragus lerviae were submitted to NCBI GenBank (accession numbers in Table 1). The phylogenetic relationships of these haplotypes with the available sequences of other Caprinae in the GenBank, Capra, Ovis and Ammotragus were investigated using the sequence of Bos taurus as outgroup. Neighbor-Joining (NJ), Maximum Parsimony (MP), Maximum-Likelihood 
(ML) or Bayesian approaches were used under different models of nucleotide substitution. All positions containing gaps were eliminated. A Neighbor-Joining (NJ) tree of haplotypes based on the simple Jukes-Cantor distance was constructed with MEGA. The reliability of the nodes was assessed by 1,000 bootstrap replicates [66]. Additionally, the optimal substitution model was determined with MEGA 5 using the Bayesian Information Criterion [67]. We used this model to analyze phylogenies both by $\mathrm{NJ}$ and Maximum Likelihood (ML) using the Close-Neighbor-Interchange method as tree searching strategy. The topology of the tree was further investigated by model free Maximum Parsimony (MP) as implemented in MEGA, using the Max-mini branchand-bound algorithm. The MP consensus tree was inferred from 1000 bootstrap replicates. Bayesian analysis was conducted using the Monte Carlo Markov Chains (MCMC) method implemented in BEAST v1.6.1 [68]. A relaxed lognormal model of lineage variation and a coalescent prior with constant size, appropriate when the alignments contain multiple intraspecific sequences, were assumed. The model of nucleotide substitution was HKI+G (also appropriate to describe the observed substitution pattern since it has the second lowest BIC score obtained with MEGA). The empirical nucleotide sequences and a gamma distribution of site heterogeneity with 5 categories of substitution rates were set as priors. Two replicates were run for 25 million generations with tree and parameter sampling every 1,000 generations. Subsequently, the sampling distributions of the two different replicates were combined using LogCombiner and the resulting samples were summarized using the software TreeAnnotator, using a burnin of 2,500 and under the maximum clade credibility option. The percentages of samples recovering any particular clade represent the clade posterior probability. Trees were visualized and edited with FigTree [69].

Divergence time between the two haplotypes of chamois was estimated with BEAST after the analysis of the sequences of Bos, Ovis, Capra and Rupicapra. The sequences of Ammotragus were not included in this analysis because of the inconsistence of its phylogenetic position that we found in the previous analysis. The conditions of the runs were as before and a relaxed uncorrelated molecular clock was employed. As calibration we used the divergence times of Bovidae (mean 25.8 mya, standard deviation [SD] 0.6 mya), Caprinae (mean 14.1 mya, SD 1.1) and Capra-Ovis (11.5 mya, SD 0.9) following Hernández-Fernández and Vrba [70] as a normal distribution prior. We placed monophyly constrains on the group Caprinae and on the groups Ovis, Capra and Rupicapra. The mean rate of nucleotide substitution and its standard deviations were obtained from the Bayesian MCMC sampled values using the program
TRACER. The analysis was repeated using the Yule speciation model as prior to check for the effects of the assumed model in the estimate. In addition, the time of divergence was also estimated using only one Ovis and one Capra sequence (five sequences in total) to check for the effects of having more than one sequence per species on the estimated time of divergence.

\section{Y microsatellite statistical analyses}

We typed 87 individuals (40 of $R$. pyrenaica and 47 of $R$. rupicapra) for the microsatellites UMN2303 and SRYM18 according to size. The SRYM18 microsatellite was found to be compound of two repeated motifs and one SNP. The SNP was treated as a binary trait and the variations in the number of repetitions of the two motifs were treated as two markers. Thus microsatellite individual genotypes were arranged in a matrix of 3 repeated motifs per 87 individuals.

Descriptive statistics analysis was performed with MSAnalyzer v3.12 [71]. We calculated for each repeated motif, in an EXCEL worksheet, the absolute difference in number of repeats (Dad) between the pair of species and its variance to check for differences associated to the length of the repeated motif.

The evolutionary relationships between the haplotypes, of the microsatellite markers alone or the combined haplotypes including binary data (one SNP on the SRY promoter and other SNP in the SRYM18 microsatellite), were analyzed by a Median-Joining network [72] constructed with NETWORK 4.6 (Fluxus Technology Ltd.). This method differs from traditional ones by allowing extant haplotypes to occupy internal nodes. The parameter $\varepsilon$ was set to zero (default) to obtain a sparse spanning network. Haplotype components were weighted $(\mathrm{w})$ according to its mutability, increasing weight for the components with lower mutation rate. The weights were as follows: SRYM18 $[\mathrm{T}] \mathrm{n}, \mathrm{w}=1$; SRYM18 [TTC]m, $\mathrm{w}=2$ and UMN2303 [TTTTG] $\mathrm{m}, \mathrm{w}$ $=3$ for the microsatellites. In the network of haplotypes including SNPs as well as microsatellite mutations, the weight for the binary data was set to $\mathrm{w}=4$.

\section{Acknowledgements}

Ana Laviada (Oviedo) provided excellent technical assistance. For collaboration and help in collecting chamois samples the authors are indebted to the following institutions: the Regional Governments of Principado de Asturias (Consejería de Agricultura) and Aragón (Diputación General de Aragón), the hunting wardens from Asturias and Aragón, Camino Real Hunting and the following people: José Francisco Quirós, Javier PérezBarbería, Carlos Nores, L. Rossi, Juan Carlos del Campo, Juan Bejar, Paloma Barracina, H. Papaioannou, M. Brown, W. Gasienica-Byrcyn, T. Skalski, J. Meana, S. Erceg, P. Veinberg, J. Badridge, Alvaro Mazón, Michal Adamec, Richard Kraft, Juan Herrero, Athanassios I. Sfougaris, Friederike Spitzenberger, Rita Lorenzini, Jacques Michallet, Natalia Martinkova and Franz Suchentrunk. The Ammotragus lervia samples were provided by Manolo Ballester and Ángel Palomares. We thank Sara de Albornoz for the correction and improvement of our English. This work was funded by grant CGL2007-64315 
from the Spanish "Ministerio de Educación y Ciencia" (FEDER support included). T. Pérez was funded by the "Gobierno del Principado de Asturias" (PCTI 2006-2009). We are also indebted to the editor and two anonymous reviewers for valuable suggestions to improve the manuscript.

\section{Author details}

'Departamento de Biología Funcional, Genética, Universidad de Oviedo, Julián Clavería 6, 33006 Oviedo, Spain. Institute of Immunology, Department of Pathobiology, University of Veterinary Medicine Vienna, Veterinaerplatz 1, A-1210 Vienna, Austria.

\section{Authors' contributions}

TP ran the bulk of the laboratory work and data collection and undertook analyses and interpretation. SEH and JA carried out aspects of the molecular lab work and manuscript composition. AD conceived and coordinated the study, analysed SRY promoter sequence and microsatellite data and wrote the paper. All authors read and approved the final manuscript.

Received: 19 May 2011 Accepted: 26 September 2011

Published: 26 September 2011

\section{References}

1. Tajima F: Evolutionary relationship of dna-sequences in finite populations. Genetics 1983, 105(2):437-460.

2. Nichols R: Gene trees and species trees are not the same. Trends in Ecology \& Evolution 2001, 16(7):358-364.

3. Petit RJ, Excoffier L: Gene flow and species delimitation. Trends Ecol Evol 2009, 24(7):386-393.

4. Pääbo S: The mosaic that is our genome. Nature 2003, 421(6921):409-412.

5. Arnold ML, Meyer A: Natural hybridization in primates: One evolutionary mechanism. Zoology 2006, 109(4):261-276

6. Arnold ML: Reticulate evolution and humans: origins and ecology. Oxford; New York: Oxford University Press; 2009.

7. Schwenk K, Brede N, Streit B: Introduction. Extent, processes and evolutionary impact of interspecific hybridization in animals. Philos Trans R Soc Lond B Biol Sci 2008, 363(1505):2805-2811.

8. Barraclough TG, Vogler AP: Detecting the geographical pattern of speciation from species-level phylogenies. American Naturalist 2000, 155(4):419-434.

9. Grubb P: Orden Artiodactila. Mammal Species of the World Washington: Smithsonian Institution Press; 1993, 374-414.

10. Lovari S: Evolutionary aspects of the biology of Chamois. Rupicapra spp. (Bovidae, Caprinae). The biology and management of capricornis and related mountain antelopes London: Croom Helm; 1987, 51-61.

11. Nascetti G, Lovari S, Lanfranchi P, Berducou C, Mattiucci S, Rossi L, Bullini L: Revision of Rupicapra genus. III. Electrophoretic studied demonstrating species distinction of chamois populations of the Alps from those of the Apennines and Pyrenees. Biology and Management of Mountain Ungulates London: Croom Helm; 1985, 56-62.

12. Pérez T, Albornoz J, Garcíavázquez E, Domínguez A: Application of DNA fingerprinting to population study of chamois (Rupicapra rupicapra). Biochemical Genetics 1996, 34(7-8):313-320

13. Hammer S, Nadlinger K, Hartl GB: Mitochondrial DNA differentiation in chamois (genus Rupicapra): Implications for taxonomy, conservation, and management. Acta Theriologica 1995, 145-155.

14. Schaschl H, Goodman SJ, Suchentrunk F: Sequence analysis of the MHC class II DRB alleles in Alpine chamois (Rupicapra r. rupicapra). Developmental and Comparative Immunology 2004, 28:265-277.

15. Alvarez-Busto J, García-Etxebarria K, Herrero J, Garin I, Jugo BM: Diversity and evolution of the Mhc-DRB1 gene in the two endemic lberian subspecies of Pyrenean chamois, Rupicapra pyrenaica. Heredity 2007 99:406-413.

16. Rodríguez F, Hammer S, Perez T, Suchentrunk F, Lorenzini R, Michallet J, Martinkova N, Albornoz J, Domínguez A: Cytochrome b Phylogeography of Chamois (Rupicapra spp.). Population Contractions, Expansions and Hybridizations Governed the Diversification of the Genus. Journal of Heredity 2009, 100(1):47-55

17. Rodríguez F, Perez T, Hammer SE, Albornoz J, Domínguez A: Integrating phylogeographic patterns of microsatellite and mtDNA divergence to infer the evolutionary history of chamois (genus Rupicapra). BMC Evol Biol 2010, 10:222
18. Avise JC, Walker D, Johns GC: Speciation durations and Pleistocene effects on vertebrate phylogeography. Proceedings Biological sciences/The Royal Society 1998, 22(265):5

19. Taberlet P, Fumagalli L, Wust-Saucy AG, Cosson JF: Comparative phylogeography and postglacial colonization routes in Europe. Molecular Ecology 1998, 7(4):453-464

20. Hewitt GM: Some genetic consequences of ice ages, and their role in divergence and speciation. Biological Journal of the Linnean Society 1996, 58(3):247-276.

21. Masini F, Lovari S: Systematics, phylogenetic-relationships, and dispersal of the chamois (rupicapra spp). Quaternary Research 1988, 30(3):339-349.

22. Lalueza-Fox C, Castresana J, Sampietro L, Marques-Bonet T, Alcover JA, Bertranpetit J: Molecular dating of caprines using ancient DNA sequences of Myotragus balearicus, an extinct endemic Balearic mammal. Bmc Evolutionary Biology 2005, 5:70.

23. Ropiquet A, Hassanin A: Molecular phylogeny of caprines (Bovidae, Antilopinae): the question of their origin and diversification during the Miocene. Journal of Zoological Systematics and Evolutionary Research 2005, 43(1):49-60.

24. Rodríguez F, Albornoz J, Domínguez A: Cytochrome b pseudogene originated from a highly divergent mitochondrial lineage in genus Rupicapra. Journal of Heredity 2007, 98(3):243-249.

25. Crestanello B, Pecchioli E, Vernesi C, Mona S, Martínková N, Janiga M, Hauffe HC, Bertorelle $\mathrm{G}$ : The Genetic Impact of Translocations and Habitat Fragmentation in Chamois (Rupicapra) spp. Journal of Heredity 2009, 100(6):691-708.

26. Pérez T, Albornoz J, Domínguez A: Phylogeography of chamois (Rupicapra spp.) inferred from microsatellites. Molecular Phylogenetics and Evolution 2002, 25(3):524-534.

27. Petit E, Balloux F, Excoffier L: Mammalian population genetics: why not Y? Trends in ecology \& evolution (Personal edition) 2002, 17(1):28-33.

28. Hurles ME, Jobling MA: Haploid chromosomes in molecular ecology: lessons from the human Y. Mol Ecol 2001, 10(7):1599-1613.

29. Perez-Pardal L, Royo L, Beja-Pereira A, Curik I, Traore A, Fernandez I, Solkner J, Alonso J, Alvarez I, Bozzi R, et al: Y-specific microsatellites reveal an African subfamily in taurine (Bos taurus) cattle. Anim Genet 2010, 41(3):232-241.

30. Tamura K: Estimation of the number of nucleotide substitutions when there are strong transition-transversion and $\mathrm{G}+\mathrm{C}$-content biases. Mol Biol Evol 1992, 9(4):678-687.

31. Meadows JR, Hanotte O, Drogemuller C, Calvo J, Godfrey R, Coltman D, Maddox JF, Marzanov N, Kantanen J, Kijas JW: Globally dispersed Y chromosomal haplotypes in wild and domestic sheep. Anim Genet 2006, 37(5):444-453.

32. Lovari S, Scala C: Revision of Rupicapra Genus. 1. A statistical reevaluation of Couturier's data on the morphometry of six chamois subspecies. Bollettino Di Zoologia 1980, 47:12.

33. Corlatti L, Lorenzini R, Lovari S: The conservation of the chamois Rupicapra spp. Mammal Review 2011, 41(2):163-174.

34. Hassanin A, Ropiquet A, Couloux A, Cruaud C: Evolution of the mitochondrial genome in mammals living at high altitude: new insights from a study of the tribe Caprini (Bovidae, Antilopinae). J Mol Evol 2009, 68(4):293-310

35. Meadows JR, Kijas JW: Re-sequencing regions of the ovine $Y$ chromosome in domestic and wild sheep reveals novel paternal haplotypes. Anim Genet 2009, 40(1):119-123.

36. Cassinello J: Ammotragus lervia: a review on systematics, biology, ecology and distribution. Annales Zoologici Fennici 1998, 35:149-162.

37. Geist V: On the evolution of the Caprinae. The biology and management of Capricornis and related mountain Antelopes London: Croom Helm London; 1987, 3-40.

38. Bunch TD, Foote WC, Spillett JJ: Translocations of acrocentric chromosomes and their implications in the evolution of sheep (Ovis). Cytogenet Cell Genet 1976, 17(3):122-136.

39. Roca AL, Georgiadis N, O'Brien SJ: Cytonuclear genomic dissociation in African elephant species. Nat Genet 2005, 37(1):96-100.

40. Johnson WE, Eizirik E, Pecon-Slattery J, Murphy WJ, Antunes A, Teeling E, O'Brien SJ: The late Miocene radiation of modern Felidae: a genetic assessment. Science 2006, 311(5757):73-77.

41. Nakagome S, Pecon-Slattery J, Masuda R: Unequal rates of $Y$ chromosome gene divergence during speciation of the family Ursidae. Mol Biol Evol 2008, 25(7):1344-1356. 
42. Xue Y, Wang Q, Long Q, Ng BL, Swerdlow H, Burton J, Skuce C, Taylor R, Abdellah Z, Zhao $Y$, et al: Human $Y$ chromosome base-substitution mutation rate measured by direct sequencing in a deep-rooting pedigree. Curr Biol 2009, 19(17):1453-1457.

43. Forster $P$, Rohl A, Lunnemann P, Brinkmann C, Zerjal T, Tyler-Smith $C$, Brinkmann B: A short tandem repeat-based phylogeny for the human $Y$ chromosome. Am J Hum Genet 2000, 67(1):182-196.

44. Ropiquet A, Hassanin A: Molecular evidence for the polyphyly of the genus Hemitragus (Mammalia, Bovidae). Molecular Phylogenetics and Evolution 2005, 36(1):154-168.

45. Jarve M, Zhivotovsky LA, Rootsi S, Help H, Rogaev El, Khusnutdinova EK, Kivisild T, Sanchez JJ: Decreased rate of evolution in Y chromosome STR loci of increased size of the repeat unit. PLoS One 2009, 4(9):e7276.

46. Vázquez JF, Pérez T, Albornoz J, Domínquez A: Estimation of microsatellite mutation rates in Drosophila Melanogaster. Genetical Research 2000, 76(3):323-326.

47. Ellegren $\mathrm{H}$ : Microsatellite mutations in the germline: implications for evolutionary inference. Trends Genet 2000, 16(12):551-558.

48. Hellborg L, Ellegren $\mathrm{H}$ : $\mathrm{Y}$ chromosome conserved anchored tagged sequences (YCATS) for the analysis of mammalian male-specific DNA Mol Ecol 2003, 12(1):283-291.

49. Charlesworth B, Charlesworth D: Rapid fixation of deleterious alleles can be caused by Muller's ratchet. Genet Res 1997, 70(1):63-73.

50. Domínguez A, Albornoz J, Santiago E: Analysis of lethals in selected lines of Drosophila melanogaster. Theoretical and Applied Genetics 1987. 74(3):409-413

51. Hellborg L, Ellegren $\mathrm{H}$ : Low levels of nucleotide diversity in mammalian $Y$ chromosomes. Mol Biol Evol 2004, 21(1):158-163.

52. Rodriguez F, Albornoz J, Domínguez A: Cytochrome B pseudogene originated from a highly divergent mitochondrial lineage in genus rupicapra. Journal of Heredity 2007, 98(3):243-249.

53. Carranza S, Arnold EN, Wade E, Fahd S: Phylogeography of the false smooth snakes, Macroprotodon (Serpentes, Colubridae): mitochondrial DNA sequences show European populations arrived recently from Northwest Africa. Mol Phylogenet Evol 2004, 33(3):523-532.

54. Martínez-Solano I, Gonçalves HA, Arntzen JW, CGarcía-París M: Phylogenetic relationships and biogeography of midwife toads (Discoglossidae: Alytes). Journal of Biogeography 2004, 31:603-618.

55. Pidancier N, Jordan S, Luikart G, Taberlet P: Evolutionary history of the genus Capra (Mammalia, Artiodactyla): discordance between mitochondrial DNA and Y-chromosome phylogenies. Mol Phylogenet Evol 2006, 40(3):739-749.

56. Ropiquet A, Gerbault-Seureau M, Deuve JL, Gilbert C, Pagacova E, Chai N, Rubes J, Hassanin A: Chromosome evolution in the subtribe Bovina (Mammalia, Bovidae): the karyotype of the Cambodian banteng (Bos javanicus birmanicus) suggests that Robertsonian translocations are related to interspecific hybridization. Chromosome Res 2008 16(8):1107-1118

57. Goodman SJ, Barton NH, Swanson G, Abernethy K, Pemberton JM: Introgression through rare hybridization: A genetic study of a hybrid zone between red and sika deer (genus Cervus) in Argyll, Scotland. Genetics 1999, 152(1):355-371.

58. Moore NW, Halnan CR, McKee JJ, Watson Jl: Studies on hybridization between a Barbary ram (Ammotragus lervia) and domestic ewes (Ovis aries) and nanny goats (Capra hircus). J Reprod Fertil 1981, 61(1):79-82.

59. Cattaneo C, Smillie DM, Gelsthorpe K, Piccinini A, Gelsthorpe AR, Sokol RJ: A simple method for extracting dna from old skeletal material. Forensic Science International 1995, 74(3):167-174.

60. Sambrook J, Fritsch EF, Maniatis T: Molecular cloning - A laboratory manual. New York: Cold Spring Habour Laboratory Press; 1989.

61. Estoup A, Largiader CR, Perrot E, Chourrout D: Rapid one-tube DNA extraction for reliable PCR detection of fish polymorphic markers and transgenes. Molecular Marine Biology and Biotechnology 1996, 5(4):295-298.

62. Ennis S, Gallagher TF: A PCR-based sex-determination assay in cattle based on the bovine amelogenin locus. Anim Genet 1994, 25(6):425-427.

63. Liu WS, Mariani P, Beattie CW, Alexander $L$, Ponce De Leon FA: A radiation hybrid map for the bovine Y Chromosome. Mamm Genome 2002, 13(6):320-326

64. Sambrook J, Fritsch E, Maniatis T: Molecular Cloning: A Laboratory Manual. New York: Cold Spring Harbor Laboratory Press; 1989.
65. Kumar S, Nei M, Dudley J, Tamura K: MEGA: A biologist-centric software for evolutionary analysis of DNA and protein sequences. Briefings in Bioinformatics 2008, 9(4):299-306.

66. Felsenstein J: Confidence-limits on phylogenies - an approach using the bootstrap. Evolution 1985, 39(4):783-791.

67. Wagner A, Lewis C, Bichsel M: A survey of bacterial insertion sequences using IScan. Nucleic Acids Res 2007, 35(16):5284-5293.

68. Drummond AJ, Rambaut A: BEAST: Bayesian evolutionary analysis by sampling trees. Bmc Evolutionary Biology 2007, 7.

69. Rambaut A: FigTree: Tree figure drawing tool, version 1.0. Institute of Evolutionary Biology, University of Edinburgh; 2006.

70. Hernandez Fernandez M, Vrba ES: A complete estimate of the phylogenetic relationships in Ruminantia: a dated species-level supertree of the extant ruminants. Biol Rev Camb Philos Soc 2005, 80(2):269-302.

71. Dieringer D, Schlötterer C: Microsatellite analyser (MSA): a platform independent analysis tool for large microsatellite data sets. Molecular Ecology Notes 2002, 3:167-169.

72. Bandelt HJ, Forster $P$, Rohl A: Median-joining networks for inferring intraspecific phylogenies. Molecular Biology and Evolution 1999, 16(1):37-48.

73. IUCN Red List of Threatened Species:[http://www.iucnredlist.org].

74. Ross DG, Bowles J, Koopman P, Lehnert S: New insights into SRY regulation through identification of $5^{\prime}$ conserved sequences. BMC Mol Biol 2008, 9:85.

75. Muraoka H, Kodama T, Nakahori Y, Nakagome Y, Tanaka S, Tojo H, Tachi C: Cloning of the Genomic SRY gene in Shiba Goat (Caprahircus var. Shiba). 1996.

doi:10.1186/1471-2148-11-272

Cite this article as: Pérez et al:: Y-chromosome phylogeny in the evolutionary net of chamois (genus Rupicapra). BMC Evolutionary Biology 2011 11:272.

\section{Submit your next manuscript to BioMed Central and take full advantage of:}

- Convenient online submission

- Thorough peer review

- No space constraints or color figure charges

- Immediate publication on acceptance

- Inclusion in PubMed, CAS, Scopus and Google Scholar

- Research which is freely available for redistribution

Submit your manuscript at www.biomedcentral.com/submit
C) Biomed Central 